\title{
Braiding non-Abelian quasiholes in fractional quantum Hall states
}

\author{
Yang-Le Wu, ${ }^{1}$ B. Estienne, ${ }^{2,3}$ N. Regnault,,${ }^{1,4}$ and B. Andrei Bernevig ${ }^{1}$ \\ ${ }^{1}$ Department of Physics, Princeton University, Princeton, New Jersey 08544, USA \\ ${ }^{2}$ Sorbonne Universités, UPMC Univ Paris 06, UMR 7589, LPTHE, F-75005, Paris, France \\ ${ }^{3}$ CNRS, UMR 7589, LPTHE, F-75005, Paris, France \\ ${ }^{4}$ Laboratoire Pierre Aigrain, ENS-CNRS UMR 8551, \\ Universités P. et M. Curie and Paris-Diderot, 24, rue Lhomond, 75231 Paris Cedex 05, France
}

(Dated: August 20, 2018)

\begin{abstract}
Quasiholes in certain fractional quantum Hall states are promising candidates for the experimental realization of non-Abelian anyons. They are assumed to be localized excitations, and to display non-Abelian statistics when sufficiently separated, but these properties have not been explicitly demonstrated except for the Moore-Read state. In this work, we apply the newly developed matrix product state technique to examine these exotic excitations. For the Moore-Read and the $\mathbb{Z}_{3}$ ReadRezayi states, we estimate the quasihole radii, and determine the correlation lengths associated with the exponential convergence of the braiding statistics. We provide the first microscopic verification for the Fibonacci nature of the $\mathbb{Z}_{3}$ Read-Rezayi quasiholes. We also present evidence for the failure of plasma screening in the nonunitary Gaffnian wave function.
\end{abstract}

PACS numbers: 05.30.Pr, 73.43.Cd, 03.67.Mn

Non-Abelian anyons have been the focus of much theoretical and experimental interest due to the exciting prospect of topologically fault-tolerant quantum computing [1]. As noted by Kitaev [1, the topological degeneracy of these exotic excitations allows nonlocal storage of quantum information, while adiabatic braiding implements unitary quantum gates. Candidates $[22$ 24. for their physical realization are the quasiholes in certain fractional quantum Hall states [25, in particular, those around the plateaus at fillings $\nu=5 / 2$ and $12 / 5$ 26-28. Their model wave functions, namely, the Moore-Read [29] (MR) and the $\mathbb{Z}_{3}$ Read-Rezayi [30] (RR) states, enjoy an elegant first-quantized rewriting [29, 31] in terms of conformal field theory [32 34] (CFT) correlators, from which many physical properties can be predicted. The strengths of this approach rest on a crucial conjecture 29]: quasihole braiding statistics can be directly read off from the monodromy of the CFT correlators. Under this conjecture, the MR quasiholes are Ising anyons, while the $\mathbb{Z}_{3} \mathrm{RR}$ ones are Fibonacci anyons. But the proof of the conjecture itself is lacking.

The relation between statistics and monodromy was originally established for the Laughlin state [35] through the plasma analogy [36]. Assuming sufficient quasihole separations, the statistics-monodromy equivalence holds true when the plasma is in the screening phase. With considerable effort, this line of argument was recently extended to the MR state 37 39], in agreement with finite-size numerics [40 42]. More complicated states like the $\mathbb{Z}_{3}$ RR still remain uncharted territory for both analytics and numerics, despite their capacity for universal quantum computation [2] 4]. Moreover, wave functions constructed from nonunitary field theories (such as the Gaffnian [43]) are conjectured not to give rise to sensible statistics 38, yet the microscopic symptom of such pathology is still under investigation [44].

In this Letter, we aim to settle the aforementioned issues through numerical studies of certain model wave functions. Until very recently, this was a daunting task due to the exponentially large Hilbert space, and in many cases, the absence of a convenient analytical form of the quasihole wave functions. In fact, so far only the Laughlin and the MR quasiholes have been tested directly, with various degrees of success, using exact diagonalization and Monte Carlo techniques [40 42. A similar check on the Gaffnian and the $\mathbb{Z}_{3} \mathrm{RR}$ states proves extremely challenging due to the combinatorial complexity of their analytical expressions. These difficulties are partially solved by the recent development 4548 of exact matrix product states [49, 50] (MPS) for the CFT-derived wave functions [29, 31. The MPS formalism provides a faithful and efficient representation of quantum Hall model states, and greatly facilitates the calculation of physical observables. We generalize this novel technique to non-Abelian quasiholes. In the following, we focus on the physical results, and leave the technical details to a forthcoming paper [51]. For the $\mathbb{Z}_{k \leq 3} \mathrm{RR}$ states (including Laughlin and MR at $k=1,2$ ), as well as the Gaffnian wave function, we construct MPS for localized quasiholes, and estimate their radii from the electron density profile.

TABLE I. Numerical data for quasihole radii $R$ and the correlation length $\xi_{\text {ortho }}$ associated with wave function orthogonality [see the discussion after Eq. [8]].

\begin{tabular}{ccccc}
\hline \hline & $\nu$ & \multicolumn{2}{c}{$R / \ell_{0}$} & $\xi_{\text {ortho } / \ell_{0}}$ \\
\hline Laughlin & $\frac{1}{3}$ & \multicolumn{2}{c}{$\frac{e}{3}: 2.6$} & - \\
\hline Moore-Read & $\frac{1}{2}$ & $\frac{e}{4}: 2.8$ & $\frac{e}{2}: 2.7$ & 2.6 \\
\hline $\mathbb{Z}_{3}$ Read-Rezayi & $\frac{3}{5}$ & $\frac{e}{5}: 3.0$ & $\frac{3 e}{5}: 2.8$ & 3.4 \\
\hline \hline
\end{tabular}




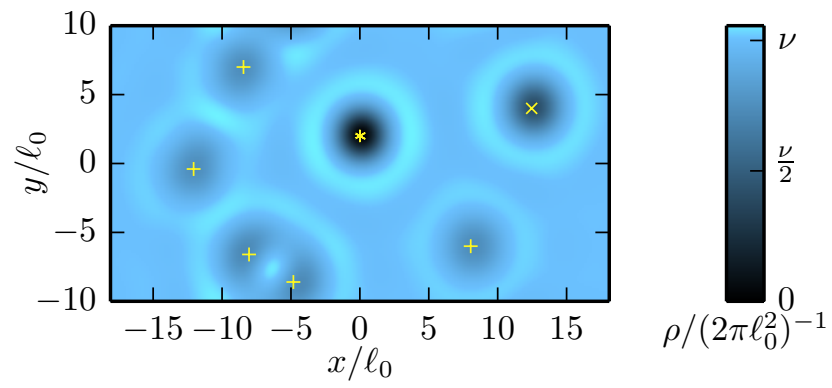

FIG. 1. Electron density for $\mathbb{Z}_{3} \mathrm{RR} \frac{e}{5}(+), \frac{2 e}{5}(\times)$ and $\frac{3 e}{5}(*)$ quasiholes on an infinite cylinder with perimeter $L_{y}=20 \ell_{0}$.

From adiabatic transport, we obtain the braiding matrices, verify the link between statistics and monodromy for $\mathbb{Z}_{k \leq 3} \mathrm{RR}$ at large quasihole separations, and determine the associated length scales. Numerical data are summarized in Table I. Through these characterizations, we confirm the Fibonacci nature of the $\mathbb{Z}_{3}$ RR quasiholes, and we provide the first size estimate for these exotic excitations. Our results also give a microscopic diagnosis for the pathology of the Gaffnian.

We consider model wave functions defined by CFT correlators [29, 31] on the cylinder geometry [52]. Evaluated in the Hamiltonian picture 45], such conformal correlators allow a MPS representation over the Landau orbitals [46, with the auxiliary space being the truncated conformal Hilbert space [53]. This truncation is constrained by the entanglement area law [54]: the auxiliary space has to grow exponentially with the cylinder perimeter $L_{y}$. Compared with previous studies [45 48, the new challenge here stems from the nontrivial fusion of non-Abelian quasiholes. Fixing all the pinned quasihole coordinates does not specify a single wave function. Rather, it defines a multidimensional vector space of degenerate states [29, 55]. A natural basis in this space arises from the conformal blocks [29], labeled by topological charges on fusion tree diagrams [33, 56]. Our construction produces a MPS for each conformal block.

We illustrate this procedure using the MR state. It is constructed 29] from the chiral Ising CFT [57], with primary fields $(\mathbb{1}, \psi, \sigma)$ and fusion rules

$$
\psi \times \psi=\mathbb{1}, \quad \psi \times \sigma=\sigma, \sigma \times \sigma=\mathbb{1}+\psi,
$$

where $\psi$ represents an electron and $\sigma$ carries a quasihole. Because only $\sigma \times \sigma$ has multiple outcomes, to enumerate $n$-quasihole states, we only need to consider the fusion trees of $n \sigma$ fields. For example, for an even number of electrons, there are two degenerate four-quasihole states,

$$
\left|\Psi_{a}\right\rangle=\underbrace{\sigma}_{a} \ell_{\sigma}^{\sigma} \ell_{\mathbb{1}}^{\sigma}, a=\mathbb{1} \text { or } \psi \text {. }
$$

The structural similarity between the above fusion tree and the usual MPS diagram [50] is not a coincidence, but
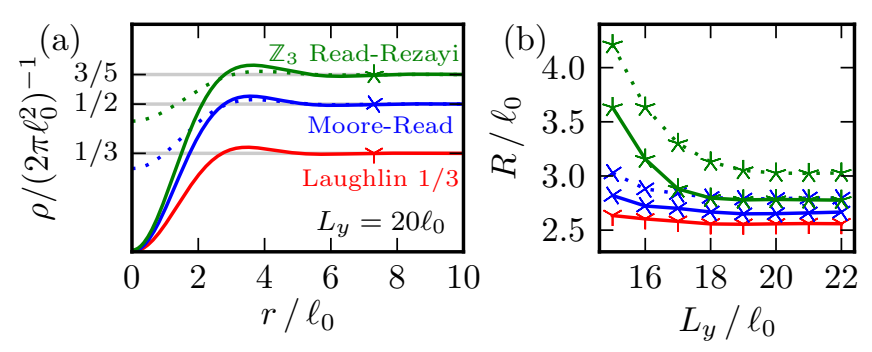

FIG. 2. (a) Radial dependence of electron density $\rho$ near a quasihole, at $L_{y}=20 \ell_{0}$. For $\mathbb{Z}_{k=2,3} \mathrm{RR}$, we study both the $\frac{e}{k+2}$ (dotted) and the $\frac{k e}{k+2}$ quasiholes (solid curves). (b) Quasihole radius $R$ as a function of cylinder perimeter $L_{y}$, using the markers defined in (a).

rather mandated by the radial ordering in the conformal correlator [51]. The $\mathbb{Z}_{3} \mathrm{RR}$ CFT has a similar structure [30, 58, with primary fields $\left(\mathbb{1}, \psi_{1}, \psi_{2}, \varepsilon, \sigma_{1}, \sigma_{2}\right)$. Electrons and quasiholes are represented by $\psi_{1}$ and $\sigma_{1}$ fields [59, respectively. There are again two fourquasihole states

$$
\left|\Psi_{a}\right\rangle=\underbrace{\sigma_{1} \sigma_{1}}_{a} \ell_{\varepsilon}^{\sigma_{1}} \ell_{\psi_{2}}^{\sigma_{1}}, \quad a=\psi_{1} \text { or } \sigma_{2} .
$$

In the following we examine the $\mathbb{Z}_{k \leq 3} \mathrm{RR}$ states at fillings $\nu=\frac{k}{k+2}$, and discuss the Gaffnian separately afterwards.

Before studying the braiding statistics, we first check whether the quasiholes are indeed localized excitations. We check the charge $\nu e=\frac{k e}{k+2}$ Abelian quasiholes, and for the $k>1$ theories, also the fundamental $\frac{e}{k+2}$ nonAbelian quasiholes. In the electron density profile, we find a localized and isotropic reduction around quasihole centers, which does not depend on the presence of other quasiholes sufficiently far away. An example is given in Fig. 1 for the $\operatorname{RR} \frac{e}{5}, \frac{2 e}{5}$ (two $\frac{e}{5}$ fused in $\sigma_{2}$ ), and $\frac{3 e}{5}$ quasiholes. The electron density displays small ripples in the periphery of a quasihole, as seen more clearly in the radial plots in Fig. 2(a). Following Ref. 60, we extract the quasihole radius $R$ from the second moment of the charge excess distribution 61. This definition is susceptible to the thin-cylinder density wave background 62, but it quickly converges as $L_{y}$ increases [Fig. 2(b)]. We note that the quasihole size barely depends on $k$. For $k>1$, the $\frac{e}{k+2}$ fundamental and the $\frac{k e}{k+2}$ Abelian quasiholes also have comparable sizes. Listed in Table I, the numerical values are consistent with those previously reported for $\mathbb{Z}_{k=1,2}$ [41, 60, 63. For the $\mathbb{Z}_{3}$ RR quasiholes, our calculation provides the first radius estimate, $R \sim 3.0 \ell_{0}$. Strictly speaking this is a lower bound, as the quasiholes in the Coulomb ground state could be larger [60].

With the localized nature of the quasiholes established, we now examine their braiding statistics. For $\mathbb{Z}_{k=2,3}$ RR states, we consider the two braids $\{12\}$ and $\{23\}$ parametrized by the mobile quasihole $\eta$, as depicted in Fig. 3. We define the Berry connection over each $\mathrm{d} \eta$ seg- 

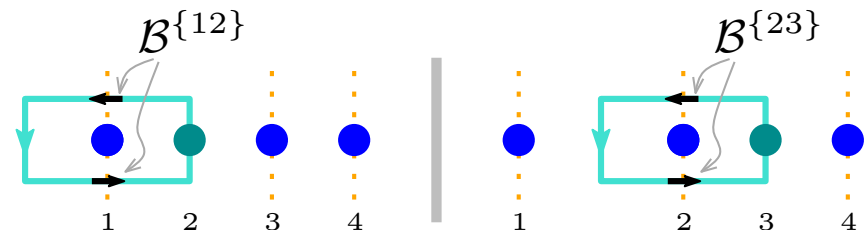

FIG. 3. Two braids of four-quasihole states, $\{12\}$ and $\{23\}$. We draw in yellow the branch cuts passing through each quasihole, and mark the half-braid $\mathcal{B}$ matrices by black arrows.

ment along the braid

$$
\mathcal{A}_{a b}(\eta ; \mathrm{d} \eta) \equiv e^{-i \mathrm{~d} \eta A_{a b}(\eta)} \equiv \frac{\left\langle\Psi_{a}(\eta+\mathrm{d} \eta) \mid \Psi_{b}(\eta)\right\rangle}{\left\|\Psi_{a}(\eta+\mathrm{d} \eta)\right\| \cdot\left\|\Psi_{b}(\eta)\right\|},
$$

in the unnormalized $\left\{\left|\Psi_{a}\right\rangle\right\}$ basis from Eqs. (2, 3). Because of the nontrivial monodromy of the CFT correlator, $\left|\Psi_{a}(\eta)\right\rangle$ is multivalued in $\eta$. At each stationary quasihole, a branch cut runs vertically around the cylinder, generating singularities in the Berry connection. If we keep $\eta$ and $\eta+\mathrm{d} \eta$ on opposite sides of the cut while letting $\mathrm{d} \eta \rightarrow 0$ [Fig. 3, the connection $\mathcal{A}(\eta ; \mathrm{d} \eta)$ tends to a constant matrix $\mathcal{B}$ not equal to the identity:

$$
\begin{aligned}
\mathcal{B}_{\mathrm{MR}}^{\{12\}} & =\left[\begin{array}{cc}
1.0 & 0 \\
0 & 1.0 i
\end{array}\right], \quad \mathcal{B}_{\mathrm{RR}}^{\{12\}}=\left[\begin{array}{cc}
e^{0.6 \pi i} & 0 \\
0 & 1.0
\end{array}\right], \\
\mathcal{B}_{\mathrm{MR}}^{\{23\}} & =\left[\begin{array}{cc}
0.5005_{5}+0.5005_{5} i & 0.4994_{6}-0.4994_{6} i \\
0.4994_{6}-0.4994_{6} i & 0.5005_{5}+0.5005_{5} i
\end{array}\right], \quad(5) \\
\mathcal{B}_{\mathrm{RR}}^{\{23\}} & =\left[\begin{array}{cc}
0.50008_{8}+0.36333_{6} i & 0.6357_{4}-0.4618_{3} i \\
0.63610_{9}-0.46215_{7} i & 0.19101_{2}+0.58786_{8} i
\end{array}\right] .
\end{aligned}
$$

These matrices virtually coincide with the half-braid matrices [33] of the CFT correlators [39, 58, as in [64]

$$
\begin{aligned}
& \underbrace{\sigma}_{a} \mathcal{L}_{\mathbb{1}}^{\sigma}=\left[\begin{array}{ll}
1 & 0 \\
0 & i
\end{array}\right]_{b a} \sum_{b}^{\sigma} \boldsymbol{L}_{\mathbb{1}}^{\sigma} \\
& =\left[\begin{array}{cc}
\frac{1+i}{2} & \frac{1-i}{2} \\
\frac{1-i}{2} & \frac{1+i}{2}
\end{array}\right]_{b a} \underbrace{\sigma}_{\sigma} \sum_{\mathbb{1}}^{\sigma} \sigma \\
& \underbrace{\sigma_{1}}_{a} \sigma_{\psi_{1}}^{\sigma_{1}} \sigma_{\psi_{1}}^{\sigma_{1}}=\left[\begin{array}{cc}
\omega^{3} & 0 \\
0 & 1
\end{array}\right]_{b a} \sum_{b}^{\sigma_{1}} \sigma_{1} \sigma_{1}^{\sigma_{1}} \sigma_{\psi_{2}}^{\sigma_{1}} \\
& =\left[\begin{array}{cc}
\Phi \omega & \sqrt{\Phi} / \omega \\
\sqrt{\Phi} / \omega & \Phi \omega^{2}
\end{array}\right]_{b a} \underbrace{\sigma_{1} \sigma_{1} \sigma_{1}}_{b} \sigma_{\psi_{2}}{ }^{\prime},
\end{aligned}
$$

with $\omega=e^{i \pi / 5}$ and $\Phi=\frac{\sqrt{5}-1}{2}$. The subscripts in Eq. (5) give the last-digit deviations from the exact values 65 .

The above half-braid $\mathcal{B}$ matrices, when squared, give the monodromy prediction for the braiding statistics, namely, Ising and Fibonacci for $\mathbb{Z}_{k=2,3} \mathrm{RR}$, respectively. These matrices should come out exactly as the CFT prediction, since we are implementing exactly the conformal blocks up to CFT truncation [53]. The actual braiding

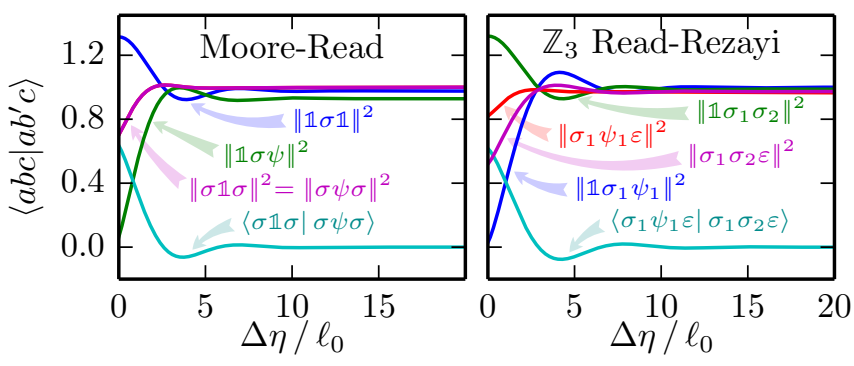

FIG. 4. Dependence of the overlaps on the quasihole separation $\Delta \eta$ at $L_{y}=20 \ell_{0} .\|a b c\|^{2}$ is shorthand for $\langle a b c \mid a b c\rangle$.

matrices are determined by the Wilson loop, which, in addition to the $\mathcal{B}$ matrices, also depends on the Berry connections away from the branch cuts. These nonsingular contributions are responsible for the potential discrepancy between monodromy and statistics, and are related to the overlap matrix $\left\langle\Psi_{a} \mid \Psi_{b}\right\rangle$ with fixed quasihole positions. As detailed in Ref. [39, if at large quasihole separation $|\Delta \eta|$, the overlap converges exponentially fast to a constant diagonal matrix,

$$
\left\langle\Psi_{a} \mid \Psi_{b}\right\rangle=C_{a} \delta_{a b}+\mathcal{O}\left(e^{-|\Delta \eta| / \xi_{a b}}\right), \text { with } C_{a} \neq 0,
$$

then, except for the branch cuts, the Berry connection vanishes up to an exponentially small correction $\mathcal{A}_{a b}(\eta) \sim \mathcal{O}\left(e^{-|\Delta \eta| / \xi_{a b}}\right)$ after subtracting the AharonovBohm phase from the background magnetic field. Hence, Eq. (8) quantifies a sufficient condition for the equivalence between monodromy and statistics for wellseparated quasiholes, without the need to integrate $\mathcal{A}_{a b}(\eta)$ by brute force. We now examine its validity for $\mathbb{Z}_{k=2,3} \mathrm{RR}$. To simplify the functional form, we keep only two quasiholes at a finite separation $\Delta \eta$ and push others to infinity. The resulting states are labeled by fusion trees

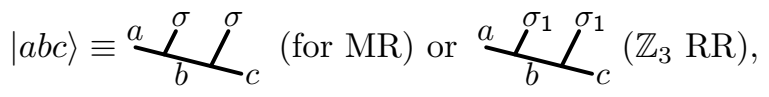

and implemented by setting the MPS boundary conditions to the leading eigenvectors of the MPS transfer matrix in topological sectors $a$ and $c$ [48. These states have the conformal-block normalization, up to a channel-independent overall constant. Plotted in Fig. 4, $\left\langle a b c \mid a b^{\prime} c\right\rangle$ indeed has the exponential convergence form of Eq. (8) in all channels. The correlation lengths can be estimated by curve fitting, or more conveniently, extracted from the spectral gaps of the transfer matrix [59. Here we focus on the length scale $\xi_{\text {ortho }}$ associated with the decaying off-diagonal elements, characterizing the orthogonality between conformal blocks, and will report the diagonal ones elsewhere [44. The numerical values are catalogued in Table I For MR, our results agree with Ref. 42]. For $\mathbb{Z}_{3} \mathrm{RR}$, combined with the $\mathcal{B}$ matrices shown earlier, the finite correlation lengths establish the quasiholes as Fibonacci anyons. At the $\nu=12 / 5$ 


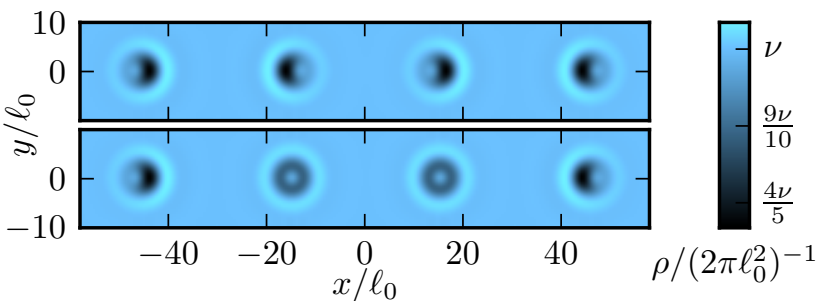

FIG. 5. Electron density for Gaffnian $\frac{e}{5}$ quasiholes in pair fusion channel $\mathbb{1}$ (top) vs. $\varphi$ (bottom) [Eq. (10] ] at $L_{y}=20 \ell_{0}$.

plateau 28, with a magnetic field of $5.4 \mathrm{~T}, \xi \sim 3.4 \ell_{0}$ translates to $0.038 \mu \mathrm{m}$. To put this in perspective, the inter-quasihole spacing in an interferometer is on the order of $0.1 \mu \mathrm{m}[66$ - the quasiholes are not quite in the well-separated regime in this case.

Finally, we go beyond the $\mathbb{Z}_{k} \mathrm{RR}$ series, and study the Gaffnian 43 wave function at filling $\nu=2 / 5$. It is derived from a nonunitary generalization of the Ising CFT, with primary fields $(\mathbb{1}, \psi, \sigma, \varphi)[\mathbf{5 9}$, and is conjectured to describe a gapless state rather than a gapped topological phase [38. There are two four-quasihole wave functions,

$$
\left|\Psi_{a}\right\rangle=\underbrace{\sigma}_{a} \gamma_{\sigma}^{\sigma} \ell_{\mathbb{1}}^{\sigma}, a=\mathbb{1} \text { or } \varphi
$$

Each fundamental quasihole has charge $e / 5$. We start from the electron density profile of these states, as shown in Fig. 5. In contrast to the $\mathbb{Z}_{k}$ RR states, the density profile here exhibits a strong fusion-channel dependence, and also a dipole-like anisotropy for $\left|\Psi_{\mathbb{1}}\right\rangle$, despite the clear separation of quasiholes. Such local distinguishability of different conformal blocks persists even when the quasiholes are infinitely separated. This casts doubt on their topological degeneracy, although it remains unclear whether the density peculiarities are genuine, or artifacts at finite cylinder perimeter $L_{y}[59$.

Unlike the unitary $\mathbb{Z}_{k}$ theories, the conformal blocks for Gaffnian are not asymptotically orthogonal in general. In place of the Berry connection, we consider the linear transform that relates $\left|\Psi_{a}(\eta)\right\rangle$ to $\left|\Psi_{b}(\eta+\mathrm{d} \eta)\right\rangle$,

$$
\frac{\left|\Psi_{b}(\eta)\right\rangle}{\left\|\Psi_{b}(\eta)\right\|}=\sum_{a} \widetilde{\mathcal{A}}_{a b}(\eta ; \mathrm{d} \eta) \frac{\left|\Psi_{a}(\eta+\mathrm{d} \eta)\right\rangle}{\left\|\Psi_{a}(\eta+\mathrm{d} \eta)\right\|}
$$

and we examine its behavior along the braids depicted in Fig. 3. Across the branch cuts, the analogue of the unitary half-braid matrices $\mathcal{B}$ are $\widetilde{\mathcal{B}} \equiv \widetilde{\mathcal{A}}(\eta ; \mathrm{d} \eta \rightarrow 0)$,

$$
\begin{aligned}
\widetilde{\mathcal{B}}^{\{12\}} & =\left[\begin{array}{cc}
e^{0.2 \pi i} & 0 \\
0 & e^{0.4 \pi i}
\end{array}\right], \\
\widetilde{\mathcal{B}}^{\{23\}} & =\left[\begin{array}{cc}
1.616_{2} & 0.746_{1}+1.028_{1} i \\
0.746_{1}+1.028_{1} i & -0.4995_{5}+1.537_{1} i
\end{array}\right],
\end{aligned}
$$

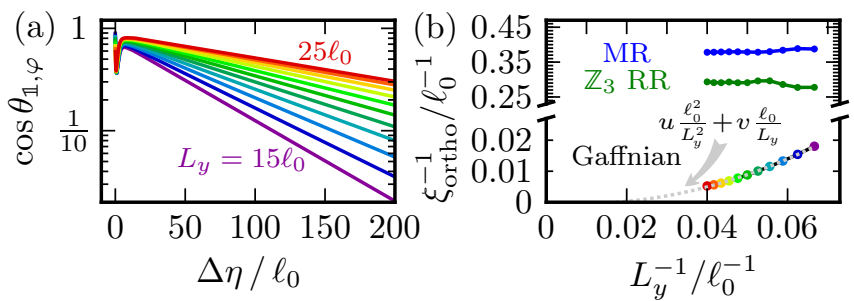

FIG. 6. (a) Asymptotic conformal-block orthogonality at large quasihole separations for Gaffnian, color-coded by $L_{y} \in\left[15\right.$.. 25] $\ell_{0}$. (b) Comparison of the $L_{y}$ dependence of the associated length scales between different theories. The Gaffnian curve has the best fit at $u=5.46(9), v=-0.095(5)$.

in agreement with the CFT prediction

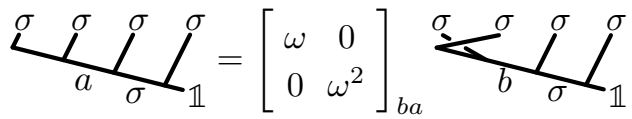

$$
\begin{aligned}
& =\left[\begin{array}{cc}
1 / \Phi & \omega^{\frac{3}{2}} / \Phi^{\frac{1}{2}} \\
\omega^{\frac{3}{2}} / \Phi^{\frac{1}{2}} & \omega^{3} / \Phi
\end{array}\right]_{b a}^{\sigma} \sum_{\sigma}^{\sigma} \sigma L_{\mathbb{1}}^{\sigma},
\end{aligned}
$$

with errors given in subscripts. Again, this agreement indicates that our MPS correctly implements the conformal blocks. The nonunitarity of $B^{\{23\}}$ comes from that of the $F_{\sigma}^{\sigma \sigma \sigma}$ matrix [67. Away from the branch cuts, the behavior of $\widetilde{\mathcal{A}}$ is again controlled by the overlaps $\left\langle\Psi_{a} \mid \Psi_{b}\right\rangle$ as a function of quasihole separations. Ideally, we would like to examine the validity of Eq. (8) for its individual matrix elements, as we do for the $\mathbb{Z}_{k} R R$ states. In the $L_{y} \lesssim 25 \ell_{0}$ regime accessible by MPS, however, this calculation is plagued by finite-size effects, and we have trouble identifying its planar limit 59. Fortunately, the conformalblock orthogonality measure $\cos \theta_{\mathbb{1}, \varphi} \equiv \frac{\langle\sigma \mathbb{1} \sigma \mid \sigma \varphi \sigma\rangle}{\|\sigma \mathbb{1} \sigma\| \cdot\|\sigma \varphi \sigma\|}$ is immune from such artifacts. Here we are keeping only two quasiholes at a finite separation $\Delta \eta$ while the outer two are set to $\pm \infty$, and we use the notation of Eq. (S3). As shown in Fig. 6. $\cos \theta_{\mathbb{1}, \varphi}$ decays exponentially as $\Delta \eta$ increases, but the associated length scale $\xi_{\text {ortho }}$ (as in $\left.\cos \theta_{\mathbb{1}, \varphi} \propto e^{-|\Delta \eta| / \xi_{\text {ortho }}}\right)$ diverges as $L_{y} \rightarrow \infty$, in sharp contrast to the $\mathbb{Z}_{k}$ RR states. Hence, in the planar limit, the conformal blocks in Eq. (10) with an untwisted tree structure acquires orthogonality extremely slowly, following a power law in $\Delta \eta$ (rather than exponentially), signaling the breakdown of the screening condition Eq. (8) for Gaffnian. The power-law behavior is consistent with the conjectured gaplessness 38, and it largely rules out the possibility of defining a sensible braiding statistics for the Gaffnian quasiholes, since the nonuniversal corrections to the monodromy matrix are not exponentially small in quasihole separations.

To summarize, in this Letter we examined numerically the quasiholes in the $\mathbb{Z}_{k \leq 3} \mathrm{RR}$ and the Gaffnian states using the MPS technique. We provide, to our knowledge, the first size estimate for the $\mathbb{Z}_{3} \mathrm{RR}$ quasiholes, and also the first microscopic, quantitative verification 
of their Fibonacci nature. We determine the correlation lengths associated with the exponential convergence of their braiding statistics. In the context of topological quantum computing, these length scales set the limit of the topological protection against decoherence in realistic systems [42, 68, 69]. Our results also shed new light on the pathology of the Gaffnian wave function manifested in its quasiholes.

Acknowledgements We thank F.D.M. Haldane, P. Bonderson, S.H. Simon, M.P. Zaletel, and S. Johri for discussions. BAB, NR, and YLW were supported by NSF CAREER DMR-095242, NSF-MRSEC DMR0819860， ONR-N00014-11-1-0635， MURI-130-6082, DARPA N66001-11-1-4110, Packard Foundation, and Keck grant. NR was also supported by the Princeton Global Scholarship. Numerical calculations were performed using the TIGRESS HPC facility at Princeton University.

[1] A. Y. Kitaev, Annals of Physics 303, 2 (2003)

[2] M. H. Freedman, M. Larsen, and Z. Wang, Communications in Mathematical Physics 227, 605 (2002)

[3] M. H. Freedman, M. J. Larsen, and Z. Wang, Communications in Mathematical Physics 228, 177 (2002).

[4] L. Hormozi, G. Zikos, N. E. Bonesteel, and S. H. Simon, Phys. Rev. B 75, 165310 (2007)

[5] C. Nayak, A. Stern, M. Freedman, and S. Das Sarma, Reviews of Modern Physics 80, 1083 (2008)

[6] M. A. Levin and X.-G. Wen, Phys. Rev. B 71, 045110 (2005).

[7] L. Fu and C. L. Kane, Phys. Rev. Lett. 100, 096407 (2008)

[8] R. M. Lutchyn, J. D. Sau, and S. Das Sarma, Phys. Rev. Lett. 105, 077001 (2010)

[9] Y. Oreg, G. Refael, and F. von Oppen, Phys. Rev. Lett. 105, 177002 (2010)

[10] J. Alicea, Y. Oreg, G. Refael, F. von Oppen, and M. P. A. Fisher, Nature Physics 7, 412 (2011).

[11] J. Alicea, Reports on Progress in Physics 75, 076501 (2012)

[12] V. Mourik, K. Zuo, S. M. Frolov, S. R. Plissard, E. P. A. M. Bakkers, and L. P. Kouwenhoven, Science 336, $1003(2012)$

[13] M. Barkeshli and X.-G. Wen, Phys. Rev. B 84, 115121 (2011)

[14] M. Barkeshli and X.-L. Qi, Physical Review X 2, 031013 (2012)

[15] M. Barkeshli, C.-M. Jian, and X.-L. Qi, Phys. Rev. B 87, 045130 (2013).

[16] D. J. Clarke, J. Alicea, and K. Shtengel, Nature Communications 4, 1348 (2013)

[17] N. H. Lindner, E. Berg, G. Refael, and A. Stern, Phys. Rev. X 2, 041002 (2012)

[18] M. Cheng, Phys. Rev. B 86, 195126 (2012)

[19] A. Vaezi, Phys. Rev. B 87, 035132 (2013).

[20] M. Barkeshli, C.-M. Jian, and X.-L. Qi, Phys. Rev. B 88, $235103(2013)$

[21] R. S. K. Mong, D. J. Clarke, J. Alicea, N. H. Lindner,
P. Fendley, C. Nayak, Y. Oreg, A. Stern, E. Berg, K. Shtengel, and M. P. A. Fisher, Phys. Rev. X 4, 011036 (2014).

[22] S. Das Sarma, M. Freedman, and C. Nayak, Phys. Rev. Lett. 94, 166802 (2005).

[23] A. Stern and B. I. Halperin, Phys. Rev. Lett. 96, 016802 (2006).

[24] P. Bonderson, K. Shtengel, and J. K. Slingerland, Phys. Rev. Lett. 97, 016401 (2006)

[25] D. C. Tsui, H. L. Stormer, and A. C. Gossard, Phys. Rev. Lett. 48, 1559 (1982)

[26] R. Willett, J. P. Eisenstein, H. L. Störmer, D. C. Tsui, A. C. Gossard, and J. H. English, Phys. Rev. Lett. 59, 1776 (1987)

[27] W. Pan, J. S. Xia, V. Shvarts, D. E. Adams, H. L. Stormer, D. C. Tsui, L. N. Pfeiffer, K. W. Baldwin, and K. W. West, Phys. Rev. Lett. 83, 3530 (1999).

[28] J. S. Xia, W. Pan, C. L. Vicente, E. D. Adams, N. S. Sullivan, H. L. Stormer, D. C. Tsui, L. N. Pfeiffer, K. W. Baldwin, and K. W. West, Phys. Rev. Lett. 93, 176809 (2004)

[29] G. Moore and N. Read, Nuclear Physics B 360, 362 (1991)

[30] N. Read and E. Rezayi, Physical Review B 59, 8084 (1999)

[31] S. Fubini, Modern Physics Letters A 06, 347 (1991).

[32] A. A. Belavin, A. M. Polyakov, and A. B. Zamolodchikov, Nuclear Physics B 241, 333 (1984)

[33] G. Moore and N. Seiberg, Communications in Mathematical Physics 123, 177 (1989).

[34] P. Di Francesco, P. Mathieu, and D. Sénéchal, Conformal Field Theory (Springer, 1999).

[35] R. B. Laughlin, Physical Review Letters 50, 1395 (1983)

[36] D. Arovas, J. R. Schrieffer, and F. Wilczek, Phys. Rev. Lett. 53, 722 (1984)

[37] V. Gurarie and C. Nayak, Nuclear Physics B 506, 685 (1997).

[38] N. Read, Phys. Rev. B 79, 045308 (2009).

[39] P. Bonderson, V. Gurarie, and C. Nayak, Physical Review B 83, 075303 (2011)

[40] Y. Tserkovnyak and S. H. Simon, Phys. Rev. Lett. 90, $016802(2003)$

[41] E. Prodan and F. D. M. Haldane, Physical Review B 80, $115121(2009)$

[42] M. Baraban, G. Zikos, N. Bonesteel, and S. H. Simon, Phys. Rev. Lett. 103, 076801 (2009)

[43 S. H. Simon, E. H. Rezayi, N. R. Cooper, and I. Berdnikov, Physical Review B 75, 075317 (2007).

[44] B. Estienne, N. Regnault, and B. A. Bernevig, ArXiv e-prints (2014), arXiv:1406.6262 [cond-mat.str-el]

[45] J. Dubail, N. Read, and E. H. Rezayi, Physical Review B 86, 245310 (2012)

[46] M. P. Zaletel and R. S. K. Mong, Physical Review B 86, 245305 (2012)

[47] B. Estienne, Z. Papic, N. Regnault, and B. A. Bernevig, Physical Review B 87, 161112 (2013).

[48] B. Estienne, N. Regnault, and B. A. Bernevig, ArXiv e-prints (2013), arXiv:1311.2936 [cond-mat.str-el]

[49] M. Fannes, B. Nachtergaele, and R. F. Werner, Communications in Mathematical Physics 144, 443 (1992)

[50] U. Schollwöck, Annals of Physics 326, 96 (2011)

[51] Y.-L. Wu, B. Estienne, N. Regnault, and B. A. Bernevig, (2014), in preparation.

[52] E. H. Rezayi and F. D. M. Haldane, Phys. Rev. B 50, 17199 (1994). 
[53] V. P. Yurov and A. B. Zamolodchikov, International Journal of Modern Physics A 05, 3221 (1990).

[54] J. Eisert, M. Cramer, and M. B. Plenio, Rev. Mod. Phys. 82, 277 (2010).

[55] C. Nayak and F. Wilczek, Nuclear Physics B 479, 529 (1996)

[56] A. Kitaev, Annals of Physics 321, 2 (2006)

[57] We leave the compactified U(1) boson implicit.

[58] E. Ardonne and K. Schoutens, Annals of Physics 322, 201 (2007)

[59] Supplemental Material.

[60] S. Johri, Z. Papić, R. N. Bhatt, and P. Schmitteckert, Phys. Rev. B 89, 115124 (2014)

[61 This definition of $R$ is very sensitive to the tail of the charge excess distribution. It clearly distinguishes quasihole sizes in different theories, even though the extent of the quasihole seems comparable between different curves in Fig. 2(a) by direct inspection.

[62] R. Tao and D. J. Thouless, Phys. Rev. B 28, 1142 (1983)

[63] M. Storni and R. H. Morf, Phys. Rev. B 83, 195306 (2011)

[64] The RR matrices here are unitarily similar to Ref. [58], due to their fusion tree choice $\psi y$ versus our $\psi<$.

[65] In the actual calculations, we push the inert quasiholes outside of the braiding loop to infinity [see Eq. (S3)]. This essentially decouples the two conformal blocks for the $\{12\}$ braid, leading to very accurate (machine precision) diagonal matrices for $\mathcal{B}^{\{12\}}$ at moderate $L_{y}$.

[66] B. Rosenow and S. H. Simon, Phys. Rev. B 85, 201302 (2012).

[67] E. Ardonne, J. Gukelberger, A. W. W. Ludwig, S. Trebst, and M. Troyer, New Journal of Physics 13, 045006 (2011)

[68] M. Cheng, R. M. Lutchyn, V. Galitski, and S. Das Sarma, Phys. Rev. Lett. 103, 107001 (2009)

[69] P. Bonderson, Phys. Rev. Lett. 103, 110403 (2009) 


\section{SUPPLEMENTAL MATERIAL}

Here we address some technical aspects of the matrix product states (MPS) for non-Abelian quasiholes derived from conformal field theory (CFT) correlators. Similar to the treatment in the main text, we will leave the compactified U(1) boson implicit in the CFT description.

\section{Fusion rules for the $\mathbb{Z}_{3}$ Read-Rezayi state}

The $\mathbb{Z}_{3}$ Read-Rezayi state can be described by the $\mathbb{Z}_{3}$ parafermion conformal field theory [S1, also known as the minimal model $\mathcal{M}(5,6)$ [S2], with central charge $c=\frac{4}{5}$. The primary fields of this CFT are $\left(\mathbb{1}, \psi_{1}, \psi_{2}, \varepsilon, \sigma_{1}, \sigma_{2}\right)$, with scaling dimensions $\left(0, \frac{2}{3}, \frac{2}{3}, \frac{2}{5}, \frac{1}{15}, \frac{1}{15}\right)$. The $\psi_{1}$ (resp. $\left.\sigma_{1}\right)$ field represents an electron (resp. a quasihole). The fusion rules of these fields are

\begin{tabular}{c|cccccc}
\hline \hline & $\mathbb{1}$ & $\psi_{1}$ & $\psi_{2}$ & $\varepsilon$ & $\sigma_{1}$ & $\sigma_{2}$ \\
\hline \hline$\psi_{1}$ & $\psi_{1}$ & $\psi_{2}$ & $\mathbb{1}$ & $\sigma_{2}$ & $\varepsilon$ & $\sigma_{1}$ \\
\hline$\sigma_{1}$ & $\sigma_{1}$ & $\varepsilon$ & $\sigma_{2}$ & $\psi_{2}+\sigma_{1}$ & $\psi_{1}+\sigma_{2}$ & $\mathbb{1}+\varepsilon$ \\
\hline \hline
\end{tabular}

\section{Fusion rules for the Gaffnian state}

The Gaffnian wave function is described $\mathrm{S3}$, by the non-unitary CFT minimal model $\mathcal{M}(3,5)$, with central charge $c=-\frac{3}{5}$. The primary fields of this CFT are $(\mathbb{1}, \psi, \sigma, \varphi)$, with scaling dimensions $\left(0, \frac{3}{4},-\frac{1}{20}, \frac{1}{5}\right)$. The $\psi$ (resp. $\sigma$ ) field represents an electron (resp. a quasihole), with fusion rules

\begin{tabular}{c|cccc}
\hline \hline & $\mathbb{1}$ & $\psi$ & $\sigma$ & $\varphi$ \\
\hline \hline$\psi$ & $\psi$ & $\mathbb{1}$ & $\varphi$ & $\sigma$ \\
\hline$\sigma$ & $\sigma$ & $\varphi$ & $\mathbb{1}+\varphi$ & $\psi+\sigma$ \\
\hline \hline
\end{tabular}

\section{The MPS transfer matrix}

Following the notation of Ref. [S4, we consider the transfer matrix $E=\sum_{m}^{0,1}\left(B^{m}\right)^{*} \otimes B^{m}$, where the $B^{m}$ matrix is associated with an empty $(m=0)$ or occupied $(m=1)$ Landau orbital in the MPS. The transfer matrix is the basic building block of any generic wave function overlap $\left\langle\Psi \mid \Psi^{\prime}\right\rangle$. It acts on a direct product of two copies of the truncated conformal Hilbert space, one copy for $\langle\Psi|$, and the other for $\left|\Psi^{\prime}\right\rangle$. From the fusion rules, we find that the CFT Hilbert space can be naturally split into two sectors, each being closed under fusion with the electron (although they are connected by fusion with the quasihole). We refer to them as the "vac" and the "qh" sectors:

\begin{tabular}{c|cc}
\hline \hline & vac & qh \\
\hline \hline Moore-Read & $\mathbb{1}, \psi$ & $\sigma$ \\
\hline $\mathbb{Z}_{3}$ Read-Rezayi & $\mathbb{1}, \psi_{1}, \psi_{2}$ & $\varepsilon, \sigma_{1}, \sigma_{2}$ \\
\hline Gaffnian & $\mathbb{1}, \psi$ & $\sigma, \varphi$ \\
\hline \hline
\end{tabular}

The $B^{m}$ matrices are block-diagonal in the sector index, $B^{m}=\bigoplus_{\alpha} B_{\alpha}^{m}$, with $\alpha$ summed over $\{$ vac, qh $\}$. Therefore, the transfer matrix is also block-diagonal,

$$
E=\bigoplus_{\alpha, \beta} E_{\alpha, \beta}, \text { with } E_{\alpha, \beta}=\sum_{m}\left(B_{\alpha}^{m}\right)^{*} \otimes B_{\beta}^{m} .
$$

We denote by $\lambda_{\alpha, \beta}^{(i)}$ the $i$-th largest eigenvalue of $E_{\alpha, \beta}$. The MPS auxiliary space is constructed from the truncated conformal Hilbert space, and the truncation is constrained by the entanglement area law. In our calculations, we have to deal with transfer matrix blocks (after various reductions [\$S4]) with dimensions as large as

\begin{tabular}{c|ccc}
\hline \hline & (vac, vac) & (vac, qh) & (qh, qh) \\
\hline \hline Moore-Read & $1.1 \times 10^{7}$ & $1.5 \times 10^{7}$ & $2.0 \times 10^{7}$ \\
\hline $\mathbb{Z}_{3}$ Read-Rezayi & $3.6 \times 10^{7}$ & $5.5 \times 10^{7}$ & $8.4 \times 10^{7}$ \\
\hline Gaffnian & $1.3 \times 10^{7}$ & $2.0 \times 10^{7}$ & $3.0 \times 10^{7}$ \\
\hline \hline
\end{tabular}

Incidentally, for the braiding and the overlap calculations, we have to work on the full direct product space without symmetry reduction, the dimension of which can be up to 25 times as large as the sizes mentioned in the previous table.

\section{Overlap calculation}

As explained in the main text, the central object in our braiding study is the overlap matrix $\left\langle a b c \mid a b^{\prime} c\right\rangle$, and we are particularly interested in its exponential convergence

$$
\left\langle a b c \mid a b^{\prime} c\right\rangle=C_{a b c} \delta_{b b^{\prime}}+\mathcal{O}\left(e^{-|\Delta \eta| / \xi_{\left\langle a b c \mid a b^{\prime} c\right\rangle}}\right) .
$$

In the following we outline the calculation of the overlap matrix using the MPS technique, and also discuss the determination of the correlation lengths. Recall from the main text that the state

$$
|a b c\rangle \equiv \frac{a}{\sigma}_{c}^{\sigma}
$$

involves two localized quasiholes at a finite separation $\Delta \eta$, and the topological charges $a$ and $c$ represent extra quasiholes pushed to the ends of the infinite cylinder. Diagrammatically, the MPS for $|a b c\rangle$ is given by [S4, S5]

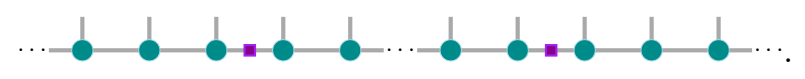


Here, the orbital $B^{m}$ matrices are represented by the green circles, with the occupation number $m=0,1$ carried by the upward-pointing leg, and the quasihole matrices are represented by the purple squares. Each quasihole matrix depends on both the quasihole position and the fusion channel context, i.e. the topological charges before and after the $\sigma$ field insertion in the fusion tree, and it is inserted into the matrix product at the correct time-ordered positions [S5. Technical details of the construction of the quasihole matrix will be addressed in a forthcoming paper [S6]. The overlap $\left\langle a b c \mid a b^{\prime} c\right\rangle$ is computed by contracting

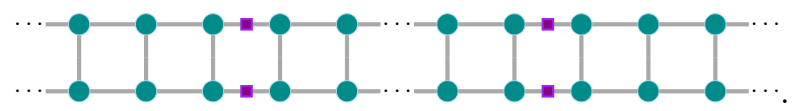

Here, the upper (lower) chain represents the $\langle a b c|\left(\left|a b^{\prime} c\right\rangle\right)$ state, respectively, and in the ladder-like structure, each rung corresponds to the transfer matrix $E$ over a single orbital [Eq. (S1)]. Although not marked explicitly in the above diagrams, the fusion channel dependence enters through the quasihole insertions (purple squares) as well as the boundary conditions. The contraction of the above tensor network can be significantly simplified on an infinite cylinder, as detailed in Ref. [S4. Essentially, an infinitely repeated action of the transfer matrix can be accurately represented by its projection into the subspace of its largest eigenvalue in the relevant sector. For the overlap $\left\langle a b c \mid a b^{\prime} c\right\rangle$ with a finite quasihole separation $\Delta \eta$, as shown in Fig. 4 of the main text, this simplification applies only to the peripheral regions outside of the two quasihole insertions. Between the two quasiholes, we have to contract the transfer matrices by brute force. However, in the limit of large $\Delta \eta$, asymptotically the overlap is still controlled by the leading few eigenmodes of the transfer matrix, and the associated correlation lengths can be simply determined from the spectral gaps of the transfer matrix, without resorting to curve fitting.

We now explain this using three representative examples. First, consider the off-diagonal element $\left\langle\sigma_{1} \psi_{1} \varepsilon \mid \sigma_{1} \sigma_{2} \varepsilon\right\rangle$ for the $\mathbb{Z}_{3}$ Read-Rezayi state. In this case, the action of the transfer matrix over the $\Delta \eta$ interval is confined to the (vac, qh) sector of the product space, while its action outside of the $\Delta \eta$ interval is purely in the (qh, qh) sector. At large $\Delta \eta$, we must have

$$
\left\langle\sigma_{1} \psi_{1} \varepsilon \mid \sigma_{1} \sigma_{2} \varepsilon\right\rangle \sim\left(\frac{\lambda_{\mathrm{vac}, \mathrm{qh}}^{(1)}}{\lambda_{\mathrm{qh}, \mathrm{qh}}^{(1)}}\right)^{\Delta \eta / \gamma}
$$

Here $\gamma=2 \pi \ell_{0}^{2} / L_{y}$ is the separation between adjacent Landau orbitals, while $\lambda_{\text {vac,qh }}^{(1)}$ and $\lambda_{\text {qh,qh }}^{(1)}$ are the largest eigenvalues of the transfer matrix in sectors (vac,qh) and

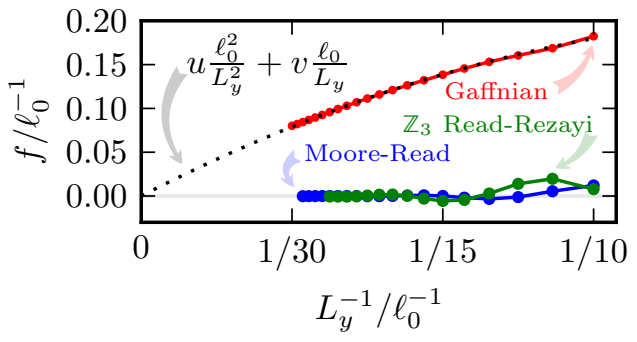

FIG. S1. Asymptotic repulsion $f$ between two plasma charges representing pinned quasiholes. The Gaffnian curve is fitted by the zero-intercept quadratic formula $f \ell_{0}=u \frac{\ell_{0}^{2}}{L_{y}^{2}}+$ $v \frac{\ell_{0}}{L_{y}}$. The best fit has $u=-8.6(2)$ and $v=2.66(1)$, with the standard error in the last digit given in parentheses.

(qh, qh), resp. The correlation length is then given by

$$
\xi_{\text {ortho }}=\left[\frac{L_{y}}{2 \pi \ell_{0}^{2}} \log \left(\frac{\lambda_{\mathrm{qh}, \mathrm{qh}}^{(1)}}{\lambda_{\text {vac,qh }}^{(1)}}\right)\right]^{-1} .
$$

As the second example, we consider the norm $\|\mathbb{1} \sigma \psi\|^{2}$ for the Moore-Read state. To the leading order, we have

$$
\|\mathbb{1} \sigma \psi\|^{2} \sim\left(\frac{\lambda_{\mathrm{qh}, \mathrm{qh}}^{(1)}}{\lambda_{\text {vac,vac }}^{(1)}}\right)^{\Delta \eta / \gamma},
$$

while $\lambda_{\text {vac,vac }}^{(1)}$ and $\lambda_{\text {qh,qh }}^{(1)}$ are the largest eigenvalues of the transfer matrix in sectors (vac,vac) and (qh, qh), resp. For $\|\mathbb{1} \sigma \psi\|^{2}$ to approach a non-zero constant value when $\Delta \eta \rightarrow \infty$ as in Eq. [S2), we need to have $\lambda_{\text {vac,vac }}^{(1)}=\lambda_{\text {qh, }}^{(1)}$. This turns out to be true for the $\mathbb{Z}_{k=2,3}$ Read-Rezayi states, up to small finite-size corrections (see below). To characterize the exponential convergence of the norm, we have to consider the second largest eigenvalue in the (qh,qh) channel, $\lambda_{\mathrm{qh}, \mathrm{qh}}^{(2)}$. The associated correlation length is given by

$$
\xi_{\mathrm{qh}}=\left[\frac{L_{y}}{2 \pi \ell_{0}^{2}} \log \left(\frac{\lambda_{\mathrm{qh}, \mathrm{qh}}^{(1)}}{\lambda_{\mathrm{qh}, \mathrm{qh}}^{(2)}}\right)\right]^{-1} .
$$

Finally, the correlation length associated with the norm $\|\sigma \psi \sigma\|^{2}$ for the Moore-Read state is similarly given by

$$
\xi_{\mathrm{vac}}=\left[\frac{L_{y}}{2 \pi \ell_{0}^{2}} \log \left(\frac{\lambda_{\mathrm{vac}, \mathrm{vac}}^{(1)}}{\lambda_{\text {vac }, \mathrm{vac}}^{(2)}}\right)\right]^{-1} .
$$

The numerical calculations of $\xi_{\text {vac }}$ and $\xi_{\text {qh }}$ are more challenging than $\xi_{\text {ortho }}$, since they depend on subleading eigenvalues of the transfer matrix. A detailed numerical study will be reported in a future paper [S7].

We now examine the $\lambda_{\text {vac,vac }}^{(1)}=\lambda_{\text {qh, qh }}^{(1)}$ condition more carefully. To have a physical understanding of its implication, we adopt the plasma analogy and reinterpret the 


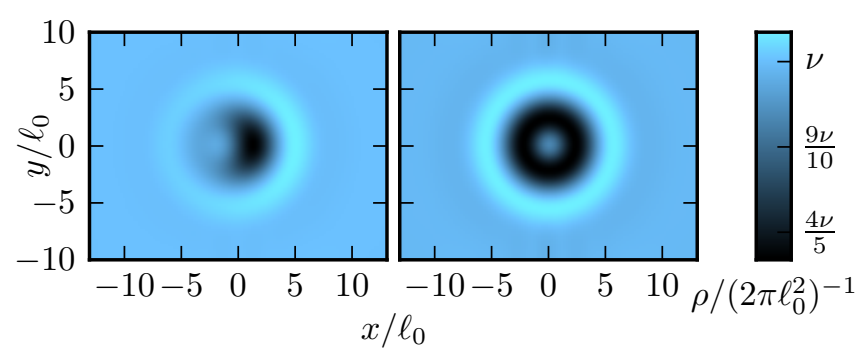

FIG. S2. Electron density around a single Gaffnian $\frac{e}{5}$ quasihole in the $|\mathbb{1} \sigma\rangle$ (left panel) and the $|\sigma \varphi\rangle$ (right panel) channels, on an infinitely long cylinder with perimeter $L_{y}=20 \ell_{0}$.

overlap in Eq. S6 as the partition function $e^{-F(\Delta \eta)}$ with two pinned charges representing the two quasiholes at a separation $\Delta \eta$. The derivative of the free energy $F(\Delta \eta)$ with respect to $\Delta \eta$ gives an effective force between the plasma charges

$$
f=-\frac{\mathrm{d} F}{\mathrm{~d} \Delta \eta} \sim \frac{L_{y}}{2 \pi \ell_{0}^{2}} \log \left(\frac{\lambda_{\mathrm{qh}, \mathrm{qh}}^{(1)}}{\lambda_{\text {vac, }, \text { vac }}^{(1)}}\right) .
$$

Therefore, if $\lambda_{\text {vac,vac }}^{(1)} \neq \lambda_{\text {qh,qh }}^{(1)}$, the two plasma charges representing quasiholes are subject to an asymptotically constant confining (if $f<0$ ) or anti-confining (if $f>0$ ) force that persists even in the limit of infinite separation. The numerical data are shown in Fig. S1. For the Moore-Read and the $\mathbb{Z}_{3}$ Read-Rezayi states, $\lambda_{\text {vac,vac }}^{(1)}$ and $\lambda_{\text {qh.qh }}^{(1)}$ quickly converge as $L_{y}$ increases. In contrast, the Gaffnian state features an asymptotic repulsion between infinitely separated plasma charges at a finite cylinder perimeter $L_{y}$, although it seems to die off in the planar limit $L_{y} \rightarrow \infty$. This makes it very hard to extract a meaningful correlation length for the diagonal elements of the overlap matrix similar to Eq. (S7). Fortunately, we can still analyze the correlation length associated with the asymptotic orthogonality of conformal blocks, as discussed in the main text.

\section{Electron density profile around Gaffnian quasiholes}

Here we show more details of the peculiarities in the electron density profile of the Gaffnian quasiholes. As noted in the main text, conformal blocks in different fusion channels are locally distinguishable despite the clear separation between quasiholes. This effect persists even when we push the quasihole separations to infinity, leaving only a single fully isolated quasihole. In this limit, the conformal blocks can be labeled by fusion tree segments

$$
|a b\rangle \equiv \stackrel{\alpha}{\sigma}_{b} .
$$

We only need to consider $|a b\rangle=|\mathbb{1} \sigma\rangle$ and $|\sigma \varphi\rangle$, since all the other possibilities can be obtained by either fusing

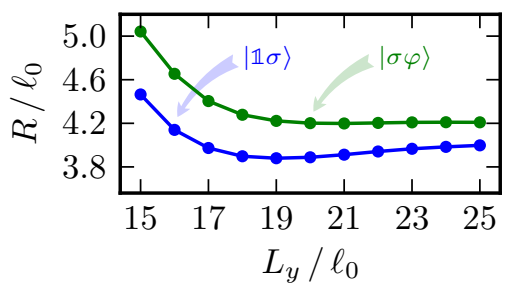

FIG. S3. Radii of Gaffnian quasiholes in $|\mathbb{1} \sigma\rangle$ and $|\sigma \varphi\rangle$ channels, as a function of the cylinder perimeter $L_{y}$.

(trivially) with $\psi$, or flipping the cylinder axis $x \rightarrow-x$. Fig. S2 shows the electron density profile for each case. The anisotropic dipole structure is clearly visible for $|\mathbb{1} \sigma\rangle$, in contrast to the isotropic $|\sigma \varphi\rangle$.

Similar to the $\mathbb{Z}_{k \leq 3}$ Read-Rezayi quasiholes analyzed in the main text, we estimate the quasihole radius from the second moment of the charge excess distribution [Fig. [S3. We have also examined the Abelian charge $\frac{2 e}{5}$ quasihole obtained by fusing two $\frac{e}{5}$ quasiholes in the $\mathbb{1}$ channel. We find a localized and isotropic density reduction around each Abelian quasihole, but this calculation turns out to be rather susceptible to the conformal Hilbert space truncation, and we have trouble reaching convergence in the radius calculation. As a final comment, we note that the peculiarities observed in the density profile are likely related to the leading eigenvalue mismatch discussed in the previous section, and are possibly artifacts at finite cylinder perimeter $L_{y}$. Unfortunately, we cannot resolve this issue using the current MPS approach, due to the fundamental constraint on $L_{y}$ from the area law of quantum entanglement.

[S1] N. Read and E. Rezayi, Physical Review B 59, 8084 (1999)

[S2] P. Di Francesco, P. Mathieu, and D. Sénéchal, Conformal Field Theory (Springer, 1999).

[S3] S. H. Simon, E. H. Rezayi, N. R. Cooper, and I. Berdnikov, Physical Review B 75, 075317 (2007).

[S4] B. Estienne, N. Regnault, and B. A. Bernevig, ArXiv e-prints (2013), arXiv:1311.2936 [cond-mat.str-el]].

[S5] M. P. Zaletel and R. S. K. Mong, Physical Review B 86, 245305 (2012)

[S6] Y.-L. Wu, B. Estienne, N. Regnault, and B. A. Bernevig, (2014), in preparation.

[S7] B. Estienne, N. Regnault, and B. A. Bernevig, (2014), unpublished. 